\title{
Upper Limits on Charm-Changing Neutral-Current Interactions
}

P. Haas, (1) M. Hempstead, (1) T. Jensen, (1) D. R. Johnson, (1) H. Kagan, (1) R. Kass, (1) P. Baringer, ${ }^{(2)}$ R. L. McIlwain, ${ }^{(2)}$ D. H. Miller, ${ }^{(2)}$ C. R. Ng, ${ }^{(2)}$ E. I. Shibata, ${ }^{(2)}$ S. Behrends, ${ }^{(3)}$ Jan M. Guida, ${ }^{(3)}$ Joan A. Guida, ${ }^{(3)}$ F. Morrow, ${ }^{(3)}$ R. Poling, ${ }^{(3)}$ E. H. Thorndike, ${ }^{(3)}$ P. Tipton, ${ }^{(3)}$ M. S. Alam, ${ }^{(4)}$ N. Katayama, ${ }^{(4)}$ I. J. Kim, ${ }^{(4)}$ W. C. Li, ${ }^{(4)}$ X. C. Lou, ${ }^{(4)}$ C. R. Sun, ${ }^{(4)}$ V. Tanikella, ${ }^{(4)}$ D. Bortoletto, ${ }^{(5)}$ M. Goldberg, ${ }^{(5)}$ R. Holmes, ${ }^{(5)}$ N. Horwitz, ${ }^{(5)}$ A. Jawahery, ${ }^{(5)}$ P. Lubrano, ${ }^{(5)}$ G. C. Moneti, ${ }^{(5)}$ V. Sharma, ${ }^{(5)}$ I. P. J. Shipsey, ${ }^{(5)}$ P. Thoma, ${ }^{(5)}$ S. E. Csorna, ${ }^{(6)}$ T. Letson, ${ }^{(6)}$ M. D. Mestayer, ${ }^{(6)}$ R. S. Panvini, ${ }^{(6)}$ G. B. Word, ${ }^{(6)}$ A. Bean, ${ }^{(7)}$ G. J. Bobbink, ${ }^{(7)}$ I. C. Brock, ${ }^{(7)}$ T. Ferguson, ${ }^{(7)}$ R. W. Kraemer, ${ }^{(7)}$ H. Vogel, ${ }^{(7)}$ C. Bebek $^{(8)}$ K. Berkelman, ${ }^{(8)}$ E. Blucher, ${ }^{(8)}$ D. G. Cassel, ${ }^{(8)}$ T. Copie, ${ }^{(8)}$ R. DeSalvo, ${ }^{(8)}$ J. W. DeWire, ${ }^{(8)}$ R. Ehrlich, ${ }^{(8)}$ R. S. Galik, ${ }^{(8)}$ M. G. D. Gilchriese, ${ }^{(8)}$ B. Gittelman, ${ }^{(8)}$ S. W. Gray, ${ }^{(8)}$ A. M. Halling, ${ }^{(8)}$ D. L. Hartill, ${ }^{(8)}$ B. K. Heltsley, ${ }^{(8)}$ S. Holzner, ${ }^{(8)}$ J. Kandaswamy, ${ }^{(8)}$ R. Kowalewski, ${ }^{(8)}$ D. L. Kreinick, ${ }^{(8)}$ Y. Kubota, ${ }^{(8)}$ N. B. Mistry, ${ }^{(8)}$ J. Mueller, ${ }^{(8)}$ R. Namjoshi, ${ }^{(8)}$ E. Nordberg, ${ }^{(8)}$ D. Perticone, ${ }^{(8)}$ D. Peterson, ${ }^{(8)}$ M. Pisharody, ${ }^{(8)}$ K. Read, ${ }^{(8)}$ D. Riley, ${ }^{(8)}$ A. Silverman, ${ }^{(8)}$ S. Stone, ${ }^{(8)}$ A. J. Sadoff, ${ }^{(9)}$ P. Avery, ${ }^{(10)}$ D. Besson, ${ }^{(10)}$ L. Garren, ${ }^{(10)}$ T. Bowcock, ${ }^{(11)}$ K. Kinoshita, ${ }^{(11)}$ F. M. Pipkin, (11) M. Procario, ${ }^{(11)}$ Richard Wilson, ${ }^{(11)}$ J. Wolinski, ${ }^{(11)}$ and D. Xiao ${ }^{(11)}$

${ }^{(1)}$ Ohio State University, Columbus, Ohio, 43210

${ }^{(2)}$ Purdue University, West Lafayette, Indiana, 47907

${ }^{(3)}$ University of Rochester, Rochester, New York 14627

${ }^{(4)}$ State University of New York at Albany, Albany, New York 12222

${ }^{(5)}$ Syracuse University, Syracuse, New York 13244

${ }^{(6)}$ Vanderbilt University, Nashville, Tennessee 37235

${ }^{(7)}$ Carnegie-Mellon University, Pittsburgh, Pennsylvania 15213

${ }^{(8)}$ Cornell University, Ithaca, New York 14853

${ }^{(9)}$ Ithaca College, Ithaca, New York 14850

${ }^{(10)}$ University of Florida, Gainesville, Florida 32611

${ }^{(11)}$ Harvard University, Cambridge, Massachusetts, 02138

(Received 23 November 1987)

\begin{abstract}
We report on a search for charm-changing neutral-current decays and lepton-family-number-nonconserving decays of charmed hadrons using inclusive and exclusive decays of charmed hadrons into oppositely charged dileptons. We find no evidence for such interactions. The upper limits on the branching ratio for inclusive charm decay to dileptons are $B\left(c \rightarrow X \mu^{+} \mu^{-}\right)<1.8 \times 10^{-2}, B\left(c \rightarrow X e^{+} e^{-}\right)$ $<2.2 \times 10^{-3}$, and $B\left(c \rightarrow X \mu^{ \pm} e^{\mp}\right)<3.7 \times 10^{-3}$ at $90 \%$ confidence level. Upper bounds are also presented for the branching ratios of several exclusive modes.
\end{abstract}

PACS numbers: $13.20 . \mathrm{Fc}, 13.40 . \mathrm{Hq}, 14.40 . \mathrm{Jz}$

In the standard electroweak theory, flavor-changing neutral-current (FCNC) interactions are explicitly forbidden in the lowest order. Historically this suppression was introduced to explain the slowness of the strangeness-nonconserving neutral-current decays $K_{L}^{0} \rightarrow \mu^{+} \mu^{-}$ and $K^{+} \rightarrow \pi^{+} v \bar{v}^{1}$ Alternative models can be constructed, however, that forbid FCNC decays of the strange and bottom quarks while allowing the existence of charm-changing neutral-current interactions. ${ }^{2,3}$ Possible consequences of such models may be large mixing in the $D^{0}-\bar{D}^{0}$ system and/or large branching ratios for charmed-hadron decays into oppositely charged leptons. Thus far, no experimental evidence exists for $D^{0}-\bar{D}^{0}$ mixing. ${ }^{4}$ Previous studies of charm-changing neutralcurrent interactions have been made by seeking the decay $D^{0} \rightarrow \mu^{+} \mu^{-}$in hadronic charm-production experiments and measurements of wrong-sign single-muon production in neutrino reactions. ${ }^{5-7}$ However, the decay
$\left(D^{0} \rightarrow \mu^{+} \mu^{-}\right)$is expected to be suppressed as a result of helicity effects. Therefore the study of inclusive charm decays to dileptons $\left(c \rightarrow X \mu^{+} \mu^{-}\right.$and $\left.c \rightarrow X e^{+} e^{-}\right)$may provide a more sensitive test of FCNC interactions of the charm quark.

Various extensions of the standard model predict the existence of massive leptoquarks which mediate leptonfamily-number-nonconserving decays such as $\mu \rightarrow e \gamma$, $K_{L}^{0} \rightarrow \mu e$, and $D^{0} \rightarrow \mu e .{ }^{8}$ Although stringent limits exist for $K_{L}^{0} \rightarrow \mu e$, certain classes of these models allow for different couplings for charge $\frac{2}{3}$ and charge $-\frac{1}{3}$ quarks, which may enhance charm decays such as $D^{0}$ $\rightarrow \mu e$. Experimental upper limits on the branching ratios for such decays provide a lower bound on the mass scale of the leptoquarks.

In this Letter, we report on a search for charmchanging neutral-current interactions and lepton-familynumber-nonconserving decays using the inclusive yield 
of $\mu^{+} \mu^{-}, e^{+} e^{-}$, and $\mu^{+} e^{-}$events in the decays of charmed hadrons produced in continuum electronpositron annihilations. We also present limits on the branching ratios for the exclusive decays $D^{0} \rightarrow \mu^{+} \mu^{-}$, $D^{0} \rightarrow e^{+} e^{-}, \quad D^{0} \rightarrow \mu^{ \pm} e^{\mp}, \quad D^{+} \rightarrow \pi^{+} \mu^{+} \mu^{-}, \quad D^{+}$ $\rightarrow \pi^{+} e^{+} e^{-}, D^{+} \rightarrow \pi^{+} \mu^{ \pm} e^{\mp}, D^{0} \rightarrow \rho^{0} \mu^{+} \mu^{-}$, and $D^{0} \rightarrow \rho^{0} e^{+} e^{-}$. Here, and throughout this Letter, charge-conjugate modes are implied.

The data sample was collected with the CLEO detector in the $Y$ energy region at the Cornell Electron Storage Ring. It consists of $77 \mathrm{pb}^{-1}$ at the $Y(4 S), 33 \mathrm{pb}^{-1}$ at the $Y(3 S)$, and $36 \mathrm{pb}^{-1}$ at energies between the $Y(3 S)$ and $\mathrm{Y}(4 S)$ resonances. The CLEO detector, the hadronic-event selection criteria, and the lepton identification procedures have been extensively described in previous publications. ${ }^{9-11}$ We identify electrons of momenta above $0.4 \mathrm{GeV} / \mathrm{c}$ using a likelihood procedure employing measurements of specific ionization $(d E / d x)$, time of flight, and electromagnetic shower energy. Our electron identification efficiency, which is limited by the solid-angle coverage of the shower detector, is a function of momentum and reaches a maximum of $40 \%$. Muons of momenta above $1.2 \mathrm{GeV} / \mathrm{c}$ are identified by their ability to penetrate an iron absorber. The muon-detection efficiency reaches a maximum of $75 \%$ for muon momenta above $1.5 \mathrm{GeV} / \mathrm{c}$. The lepton misidentification probabilities are estimated by the study of hadronic events at the $Y(1 S)$ and by the use of identified pions and protons from $K_{S} \rightarrow \pi^{+} \pi^{-}$and $\Lambda \rightarrow p \pi^{-}$decays. The threegluon decays of the $Y(1 S)$ are not expected to yield leptons. Thus, the number of charged tracks passing the lepton identification criteria from $Y(1 S)$ can be used to determine the probability that a hadron will be misidentified as a lepton. The misidentification probabilities for the criteria used in this analysis vary with the lepton momenta and range from $0.5 \%$ to $1.3 \%$ for electrons and from $0.5 \%$ to $1.7 \%$ for muons.

We study the exclusive decays listed above by searching for an enhancement at the $D^{0}$ or $D^{+}$mass in the invariant-mass spectrum of the $D$-candidate track combinations. In constructing the $D$-candidate combinations, we require that at least one of the participating tracks be identified as an electron or a muon. The lepton is combined with one (two) other tracks in the event to form all possible $D^{0}\left(D^{+}\right)$candidates. Since the combinatorial background is most serious for low momenta, where the continuum production of charm is small, ${ }^{12}$ we use only the combinations with momenta greater than $2.5 \mathrm{GeV} / \mathrm{c}$. For decays $D^{0} \rightarrow \rho^{0} \mu^{+} \mu^{-}$and $D^{0}$ $\rightarrow \rho^{0} e^{+} e^{-}$we use the decay chain $D^{*+} \rightarrow D^{0} \pi^{+}$ which, because of the excellent resolution in the measured mass difference $\left[m\left(D^{*+}\right)-m\left(D^{0}\right)\right]$, results in a significant reduction in the the combinatorial background. We define $\rho^{0}$ candidates as any two oppositely charged tracks whose invariant mass, calculated by assigning the pion mass to both tracks, is within $120 \mathrm{MeV}$

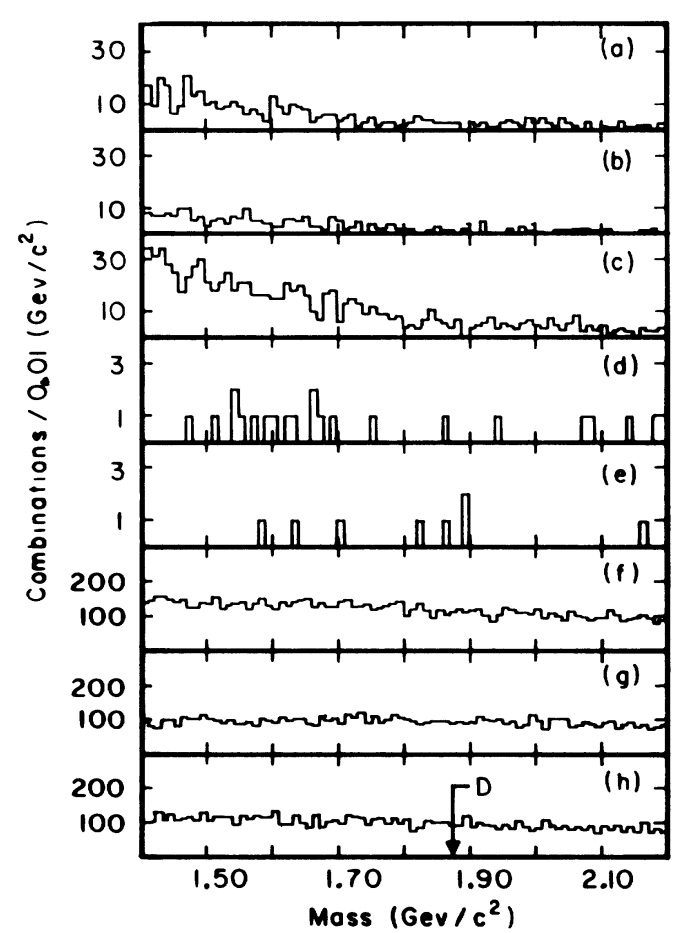

FIG. 1. Invariant-mass spectrum of $D$ candidates in (a) $D^{0} \rightarrow e^{+} e^{-}$, (b) $D^{0} \rightarrow \mu^{+} \mu^{-}$, (c) $D^{0} \rightarrow e^{ \pm} \mu^{\mp}$, (d) $D^{0}$ $\rightarrow \rho^{0} e^{+} e^{-}$, (e) $D^{0} \rightarrow \rho^{0} \mu^{+} \mu^{-}$, (f) $D^{+} \rightarrow \pi^{+} e^{+} e^{-}$, (g) $D^{+}$ $\rightarrow \pi^{+} \mu^{+} \mu^{-}$, and (h) $D^{+} \rightarrow \pi^{+} e^{ \pm} \mu^{\mp}$ modes.

of the $\rho^{0}$ mass. The invariant-mass spectra of $D$ candidates are shown in Fig. 1. Each mass spectrum is fitted by a polynomial background and a Gaussian distribution representing the $D$ signal with a mass and width predicted by a Monte Carol simulation of the $D$ decays in the detector. No significant signal is found in any of the above decay modes. The results are given in Table I.

To obtain upper limits on the exclusive modes, we normalize to the well-established decay modes $D^{0}$ $\rightarrow K^{-} \pi^{+}, D^{+} \rightarrow K^{-} \pi^{+} \pi^{+}$, and $D^{*+} \rightarrow D^{0} \pi^{+}$. Using the procedures described in Rev. 12, we find 2413 $\pm 139 D^{0} \rightarrow K^{-} \pi^{+}, 1652 \pm 300 D^{+} \rightarrow K^{-} \pi^{+} \pi^{+}$, and $636 \pm 33 D^{*+} \rightarrow D^{0} \pi^{+} \rightarrow K^{-} \pi^{+} \pi^{+}$decays, with detection efficiencies of $55 \%, 33 \%$, and $36 \%$, respectively. For example we compute the upper limit on the branching ratio for the decay $D^{0} \rightarrow \mu^{+} \mu^{-}$using

$$
\begin{aligned}
& B\left(D^{0} \rightarrow \mu^{+} \mu^{-}\right)=\frac{N\left(\mu^{+} \mu^{-}\right)}{N\left(K^{-} \pi^{+}\right)} \frac{\epsilon\left(K^{-} \pi^{+}\right)}{\epsilon\left(\mu^{+} \mu^{-}\right)} \\
& \times B\left(D^{0} \rightarrow K^{-} \pi^{+}\right),
\end{aligned}
$$

where $N\left(\mu^{+} \mu^{-}\right)$and $N\left(K^{-} \pi^{+}\right)$are the numbers of observed events, and $\epsilon\left(\mu^{+} \mu^{-}\right)$and $\epsilon\left(K^{-} \pi^{+}\right)$the detection efficiencies for the decay modes $D^{0} \rightarrow \mu^{+} \mu^{-}$and $D^{0} \rightarrow K^{-} \pi^{+}$, respectively. We use the most recent measurements of the branching ratios, $B\left(D^{0} \rightarrow K^{-} \pi^{+}\right)$ $=(4.2 \pm 0.4 \pm 0.4) \%$ and $B\left(D^{+} \rightarrow K^{-} \pi^{+} \pi^{+}\right)=(9.1$ 
TABLE I. 90\%-confidence-level upper limit on exclusive charm decays to dileptons.

\begin{tabular}{lccc}
\hline \hline Decay mode & $\begin{array}{c}\text { Upper limit on } \\
\text { number of events } \\
\text { at } 90 \% \text { confidence } \\
\text { level }\end{array}$ & $\begin{array}{c}\text { Efficiency } \\
(\%)\end{array}$ & $\begin{array}{c}\text { Upper limit on } \\
\text { branching ratio }\end{array}$ \\
\hline$D^{0} \rightarrow e^{+} e^{-}$ & 8 & 36 & $2.2 \times 10^{-4}$ \\
$D^{0} \rightarrow \mu^{+} \mu^{-}$ & 5 & 36 & $1.4 \times 10^{-4}$ \\
$D^{0} \rightarrow e^{+} \mu^{-}$ & 9 & 33 & $2.7 \times 10^{-4}$ \\
$D^{+} \rightarrow \pi^{+} e^{+} e^{-}$ & 39 & 28 & $2.6 \times 10^{-3}$ \\
$D^{+} \rightarrow \pi^{+} \mu^{+} \mu^{-}$ & 36 & 24 & $3.9 \times 10^{-3}$ \\
$D^{+} \rightarrow \pi^{+} e^{+} \mu^{-}$ & 58 & 28 & $4.5 \times 10^{-3}$ \\
$D^{0} \rightarrow \rho^{0} e^{+} e^{-}$ & 2 & 10 & $8.1 \times 10^{-4}$ \\
$D^{0} \rightarrow \rho^{0} \mu^{+} \mu^{-}$ & 5 & 4 & \\
\hline \hline
\end{tabular}

$\pm 1.3 \pm 0.4) \% .^{13}$ A similar method is used to compute the upper limits for the other modes in Table $I$. The upper bounds on the decay modes $D^{0} \rightarrow \mu^{+} \mu^{-}$and $D^{0} \rightarrow \mu^{ \pm} e^{\bar{F}}$ are comparable with previously reported limits. ${ }^{5,6,14}$ The other limits presented in Table I are first published results.

The search for the inclusive decays $c \rightarrow X \mu{ }^{ \pm} \mu^{\mp}$, $c \rightarrow X e^{ \pm} e^{\mp}$, and $c \rightarrow X \mu \pm e^{\mp}$ is performed by our measuring the dilepton yields in the continuum electronpositron annihilation data. Since the lepton yield from noncharm sources in the continuum is negligible, we look for an excess of dileptons over the expected yield from semileptonic charm decays. In order to avoid contamination from $Y$-resonance-related lepton production, we restrict this analysis to the data sample collected at the energies between the $Y(3 S)$ and $Y(4 S)$ resonances. Using our measured continuum hadronic cross section $(3.33 \pm 0.05 \mathrm{nb})^{15}$ and assuming the naive quark-model prediction that $\frac{4}{10}$ of the cross section is from charm production, we estimate that our data sample includes $47088 \pm 750$ charm events.

Candidate dilepton events are hadronic events containing two tracks identified as electrons or muons. The major background comes from "parallel decays" where two charmed particles decay semileptonically, and "fakes," where one or both candidate lepton tracks are misidentified hadrons. Parallel decays tend to produce lep- tons back to back, whereas real FCNC decays will produce leptons in one hemisphere. Hence we demand that $\cos \theta_{l l}>0.0$, where $\theta_{l l}$ is the opening angle between the leptons. To suppress photon conversions we require $\cos \theta_{l l}<0.98$. The observed numbers of dilepton events passing these criteria are given in Table II.

It is necessary to estimate and subtract the remaining contamination from the data sample. The expected numbers of fake dileptons are given in Table II. For parallel decays, a Monte Carlo simulation was done with the well-known $D^{0}$ fragmentation function and semileptonic decay spectrum ${ }^{16}$ to approximate these distributions for all charmed particles. The total number of dileptons from parallel decays can be computed with

$$
N_{l l}^{P}=N_{c c} \epsilon\left(l^{+} l^{-}\right) B\left(c \rightarrow l^{-} X\right)^{2},
$$

where $N_{c c}$ is the number of $c \bar{c}$ events, $\epsilon\left(l^{+} l^{-}\right)$is the detection efficiency for parallel decays, and $B\left(c \rightarrow l^{-} X\right)$ is the average semileptonic branching ratio of the charmed hadrons in the continuum. The latter is measured to be $(8.2 \pm 0.9) \%$, from the yield of single electrons in the continuum data. ${ }^{17}$ We predict the number of parallel-decay dileptons shown in Table II. The main uncertainties in these estimates come from the differences in the fragmentation functions, the semileptonic branching ratios, and the shapes of lepton momentum spectra of the various charmed-hadron species. Howev-

TABLE II. 90\%-confidence upper limit on inclusive charm decays to dileptons.

\begin{tabular}{lccc}
\hline \hline & $e^{ \pm} e^{\mp}$ & $\mu^{ \pm} \mu^{\mp}$ & $e^{ \pm} \mu^{\mp}$ \\
\hline $\begin{array}{l}\text { Observed } \\
\text { Expected hadronic } \\
\text { fakes }\end{array}$ & 3.0 & 1.0 & 2.0 \\
$\begin{array}{l}\text { Expected parallel } \\
\text { decays }\end{array}$ & $2.01 \pm 0.6$ & $0.7 \pm 0.2$ & $1.7 \pm 0.4$ \\
$\begin{array}{l}\text { Net excess } \\
\begin{array}{l}\text { FCNC } \\
\text { 90\% upper limit } \\
\text { on branching ratio }\end{array}\end{array}$ & $0.8 \pm 0.15$ & $0.0 \pm 0.2$ & $0.1 \pm 0.2$ \\
\hline \hline
\end{tabular}


er, after the restriction on lepton opening angle, this background is small enough that the effect of these uncertainties on the final result is negligible.

Table II shows the net number of dileptons after the subtraction of background events from parallel decays and fake dileptons. Note that FCNC decays would be expected to show up in the $e^{+} e^{-}$and $\mu^{+} \mu^{-}$channels, whereas lepton-family-number-nonconserving decays could appear as $e^{ \pm} \mu^{\mp}$ events. Clearly no significant excess of events is evident. In order to compute an upper limit on the rate for inclusive charmed-hadron decays into dileptons $\mathrm{H}_{c} \rightarrow \mathrm{Xl}^{+} \mathrm{l}^{-}$, we need to correct for the detection efficiency of such processes. We use a spectator model of the charmed-hadron decay to simulate the decay $\mathrm{H}_{c} \rightarrow \mathrm{Xl}^{+} \mathrm{l}^{-}$in the CLEO detector. In this formulation the leptonic current has a standard-model neutral-current structure. For the hadronic FCNC current we studied five possible Lorentz structures: vector $(V)$, axial vector $(A), V-A, V+A$, and constant. We find that the detection efficiency is insensitive to the parameters of the model, primarily because the smearing resulting from the Lorentz boost to the laboratory frame dominates over the small model dependence of the lepton momentum spectrum and the distribution of $\cos \theta_{l l}$. Using the efficiencies in Table II and the net number of dilepton events, we compute $90 \%$-confidence-level upper limits on the branching ratios for charm-changing neutral-current decays (Table II). For the leptonfamily-number-nonconserving decay $c \rightarrow X \mu \pm e^{\mp}$, we use a constant matrix element to estimate the detection efficiencies.

In conclusion, we have searched for flavor-changing neutral-current decays and lepton-family-numbernonconserving decays of charmed hadrons by using several exclusive decays and the inclusive dilepton yield in continuum charmed-hadron production. No evidence is found for charm-changing neutral-current interactions or lepton-family-number-nonconserving decays. We report for the first time limits on the inclusive charm decay to dileptons.
We gratefully acknowledge the effort of the Cornell Electron Storage Ring staff. Thanks are also due to the U.S. Department of Energy Outstanding Junior Investigator program (H.K. and R. Kass). This research was partially conducted with use of the Cornell National Supercomputer Facility, which is funded in part by the National Science Foundation, New York State, and IBM. This work was funded by both the National Science Foundation and the U.S. Department of Energy, under Contracts No. DE-AC02-76ER01428, No. DeAC02-76ER03066, No. De-AC02-76ER03064, No. DE-AC02-76ER01545, No. DE-AC02-78ER05001, and No. FG05-86-ER40272.

${ }^{1}$ S. L. Glashow, J. Iliopoulos, and L. Maiani, Phys. Rev. D 2, 1285 (1970).

${ }^{2}$ S. L. Glashow and S. Weinberg, Phys. Rev. D 15, 1958 (1977).

${ }^{3}$ E. A. Paschos, Phys. Rev. D 15, 1966 (1977).

${ }^{4}$ D. G. Hitlin, in Proceedings of the Internatioal Symposium on Lepton and Photon Interactions at High Energies, Hamburg, West Germany, 1987 (to be published).

5 J. J. Aubert et al., Phys. Lett. 155B, 461 (1985).

${ }^{6}$ W. C. Louis et al., Phys. Rev. Lett. 56, 1027 (1986).

${ }^{7}$ M. Holder et al., Phys. Lett. 74B, 277 (1978).

${ }^{8}$ I. I. Bigi, G. Kopp, and P. M. Zerwas, Phys. Lett. 166B, 238 (1986); W. Buchmuller and D. Wyler, Phys. Lett. B 177, 377 (1986).

${ }^{9}$ D. Andrews et al., Nucl. Instrum. Methods 211, 47 (1983).

${ }^{10}$ R. Giles et al., Phys. Rev. D 30, 2279 (1984).

${ }^{11}$ K. Chadwick et al., Phys. Rev. D 27, 475 (1983).

${ }^{12}$ D. Bortoletto et al., Phys. Rev. D 35, 19 (1987).

${ }^{13} \mathrm{~J}$. Adler et al. (MARK III Collaboration), Phys. Rev. Lett. 60, 89 (1988).

${ }^{14}$ J. J. Becker et al., Phys. Lett. B 193, 147 (1987).

${ }^{15}$ R. Giles et al., Phys. Rev. D 29, 1285 (1984).

${ }^{16}$ P. Avery et al., Phys. Rev. Lett. 51, 1139 (1983).

${ }^{17} \mathrm{~T}$. Bowcock et al., Cornell University Report No. CLNS 87-109, 1987 (to be published). 